\title{
Signatures of Random Matrix Theory in the Discrete Energy Spectra of Subnanosize Metallic Clusters
}

\author{
L. L. A. Adams*, B. W. Lang, Yu Chen and A. M. Goldman \\ School of Physics and Astronomy, University of Minnesota, \\ 116 Church St. SE, Minneapolis, MN 55455, USA and \\ ${ }^{*}$ Current address: The James Franck Institute, \\ The University of Chicago, 929 East 57th St., Chicago, IL 60637 \\ (Received textdate; Revised text)
}

\begin{abstract}
Lead clusters deposited on $\mathrm{Si}(111)$ substrates have been studied at low temperatures using scanning tunneling microscopy and spectroscopy. The current-voltage characteristics exhibit current peaks that are irregularly spaced and varied in height. The statistics of the distribution of peak heights and spacings are in agreement with random matrix theory for several clusters. The distributions have also been studied as a function of cluster shape. $\mathrm{TEX}_{\mathrm{X}}$.
\end{abstract}

\section{INTRODUCTION}

In the nearly 10 years that have passed since the first observation of discrete energy levels in metallic clusters there still remains the question of how the levels are statistically distributed in these systems. It has been suggested that random matrix theory $(\mathrm{RMT})^{1}$ is applicable to the statistical properties of the spectra of metallic clusters in much the same way that it is applicable to the slow neutron resonant spectra ${ }^{2}$ observed in the $1950 \mathrm{~s}$ and 1960s. However experimental verification of the applicability of RMT in these systems is still challenging because of the difficulty in gathering a sufficient number of levels to analyze their statistical distribution.

The first observations of discrete energy levels or "particle in a box energy levels" in metallic clusters were made by Ralph, Black and Tinkham ${ }^{3}$ in 1995 . The energy levels were observed as irregular steps contained within the Coulomb staircase in the current-voltage characteristics of clusters that were fabricated using a fixed tunneling geometry with metallic electrodes. These uneven steps in the current-voltage measurements might be a consequence of random matrix theory (RMT) $\underline{\underline{4}}$ This suggestion arises from earlier predictions that address these systems from various theoretical standpoints, including Efetov's supersymmetry derivation $\frac{\sqrt{3}}{}$ While these expectations are theoretically well established they are experimentally difficult to realize because of non-equilibrium effects $\underline{\underline{6}}$ and capacitive charging energy terms that have a tendency to mask the energy levels in mesoscopic systems. Subsequent tunneling experiments have been performed on metallic clusters ${ }^{7}$ and semiconducting $\operatorname{dots}^{8}, \underline{9}$ which have yielded results similar to those of Ralph et al., although the nature of the level statistics still remains elusive.

In contrast to metallic clusters, experimental work has exhaustively addressed the issue of distributions of level spacings and eigenfunctions of quantum dots fabricated from two dimensional electron gas systems of various predefined shapes $\frac{10}{\underline{10}}$ In these systems, electron-electron in- teractions dominate transport and the level spacing distributions appear to be Gaussian, while the distributions of the amplitudes of the eigenfunctions follow a PorterThomas distribution which is a signature of random matrix theory (RMT).

Random matrix theory (RMT) and quantum chaos were merged in the conjecture put forward by Bohigas, Giannoni and Schmit $\underline{11}$ in 1984. This conjecture states that the nearest neighbor energy level spacings of classically chaotic systems should be distributed according the Gaussian Orthogonal Ensemble (GOE), or WignerDyson $\frac{12}{1}$ distribution and this conjecture is strongly supported by aggregated numerical studies.

The Wigner-Dyson distribution which describes the statistical distribution of nearest neighbor energy levels normalized to the mean energy level has several important features as described by Porter $\underline{\underline{13}}$ First, the probability of having nearest neighbors with zero spacing disappears. Second, the probability of a level spacing is linear in energy before approaching a maximum, with the maximum occurring close to the mean energy and the tail of the distribution being fairly small. This is in contrast with completely random levels (a classically non-chaotic system) where the distribution is Poissonian. For the latter distribution the probability is largest at zero level spacing. The absence of small spacings in the Wigner-Dyson distribution is known as the: "repulsion of energy levels" $\underline{13}$ This is the key ingredient of a WignerDyson distribution and distinguishes classically chaotic from classically non-chaotic systems.

Besides the distributions of the eigenvalues which are most often addressed experimentally, it is also possible to study the statistics of the amplitudes of the eigenfunctions within the context of RMT. The corresponding distribution is called the Porter-Thomas $\frac{13}{13}$ distribution. The Porter-Thomas distribution is simply a statement about the amplitude $\psi$ of a wavefunction at any given point is a random variable. The distribution of the square of a random variable, $|\psi|^{2}$, which is Gaussian distributed, is the Porter-Thomas distribution. 
It is also interesting to note that it is possible, in some systems, to tune a transition from regular to chaotic behavior in which the distributions of the nearest neighbor level spacing change from Poisson to Wigner-Dyson. This has been found numerically in the study of the Rydberg levels of the hydrogen atom $\frac{14}{14}$ in which the transition is tuned by the application of an increasingly strong magnetic field and experimentally in diamagnetic helium by tuning the excitation energy in the presence of a magnetic field $\stackrel{15}{\underline{n}}$

Here we present experimental results that indicate that statistical distributions of highly irregularly shaped $\mathrm{Pb}$ clusters follow RMT. This is an extension of earlier work ${ }^{16}$ and its purpose is to address in detail experimental issues and present additional results which led to our conclusions. The present paper is divided into five major sections as follows: in Section II we treat the device geometry that was configured to study the discrete energy level spectra of metallic clusters. In Section III, we relate energy spectra to the geometry and discuss in detail the observed features of the spectra. In Section IV we discuss the results of the preceding section in terms of statistical distributions of both the eigenvalues and the amplitude of the moduli of the eigenfunctions. In Section $\mathrm{V}$, we demonstrate the use of scanning tunneling spectroscopy to resolve real time images of a quantity that is proportional to the square of the amplitude of the eigenfunctions in these systems.

\section{DEVICE GEOMETRY}

Before describing the current-voltage characteristics, it is worthwhile to summarize some of the salient features of the device configuration relevant to this work that are different from other spectroscopy measurements on metallic clusters. The tunneling geometry that was used is different from that used previously in three important respects. First, $\mathrm{Pb}$ clusters were grown on a semiconducting substrate. This substrate was highly resistive with a resistivity at room temperature greater than 1000 $\Omega \cdot \mathrm{cm} .17$ Second, the clusters were fabricated using the buffer layer assisted growth technique that was developed by Weaver and co-workers ${ }^{18}$ in which $\mathrm{Xe}$, an inert gas, is used as a buffer layer to control the size distribution of the clusters. This physical technique allows the clusters to land softly onto the substrate and involves no use of chemicals or organics in the growth process. Third, fabrication of the clusters and spectroscopic measurements were carried out in situ in an ultrahigh vacuum environment thus preventing contamination.

From the standpoint of conventional scanning tunneling microscopy (STM) experiments it is also unique in both the use of a highly resistive substrate and the addition of a bilayer of Ti/Pt electrodes on top of the substrate. The device geometry is illustrated in Fig. 1, The first barrier separating the STM tip (not shown) from the cluster is a vacuum barrier while the second barrier

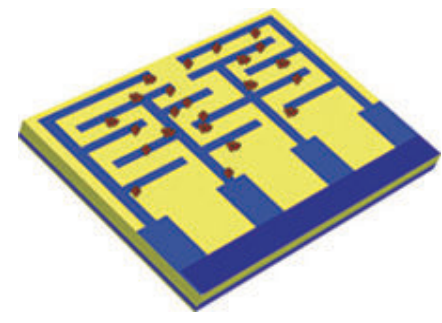

FIG. 1: An illustrative drawing of the device geometry. Electrodes were predeposited on the top of the Si substrate in the configuration shown in the diagram and contacted to the sample plate. On the back side of the $500 \mu m$ thick Si substrate, a uniform bilayer of $\mathrm{Ti} / \mathrm{Pt}$ was deposited for electrical contact to the sample plate.

between the cluster and the substrate is a thin silicon oxide barrier. This double tunnel junction arrangement is necessary for the resolution of discrete energy levels in these nanostructures. The experimental details of the cluster fabrication have been published elsewhere $\stackrel{19}{\underline{19}}$ The buffer layered assisted growth involves initially depositing four monolayers of Xe onto a cold substrate that is held at temperatures less than $50 \mathrm{~K}$. The pressure of the $\mathrm{Xe}$ is controlled upon entry into the deposition chamber by a capacitance manometer. The condensed $\mathrm{Xe}$ is then subjected to a precisely controlled exposure of $\mathrm{Pb}$ vapor flux. The average film thickness of $\mathrm{Pb}$ was less than $0.2 \AA$ as measured by a calibrated quartz crystal oscillator. Subsequently the substrate is slowly warmed to room temperature such that the Xe desorbs and the $\mathrm{Pb}$ clusters softly land onto the substrate. The resulting sample which is held on a rotable liquid helium cooled transfer rod is moved in situ into the STM chamber through a large gate valve and placed via a wobble stick onto the STM stage. All STM measurements were made with the STM operating at $4.2 \mathrm{~K}$ although the electron temperature is significantly larger. The STM tip that was used in the experiment was made from tungsten wire. The tip was characterized prior to making spectroscopy measurements by demonstrating atomic scale resolution of a graphite surface.

After fabrication, the $\mathrm{Pb}$ clusters were characteristized using STM. The heights of the clusters were measured and found to be mostly between $3 \AA$ to $12 \AA$, with an average cluster height of $8 \AA$. When a bias voltage was applied between the tip and counter-electrodes on top of the Si substrate with the cluster in between, the current was measured through the cluster. Current flows when the levels of the cluster come into juxtaposition with the level(s) at the interface state or another set of discrete energy levels from a neighboring cluster. The peaks observed in the current-voltage curves (Fig. 2) are indicative that resonant tunneling processes are operative whenever one of the cluster's quantized states is probed. This will be further elaborated upon in the next section. 


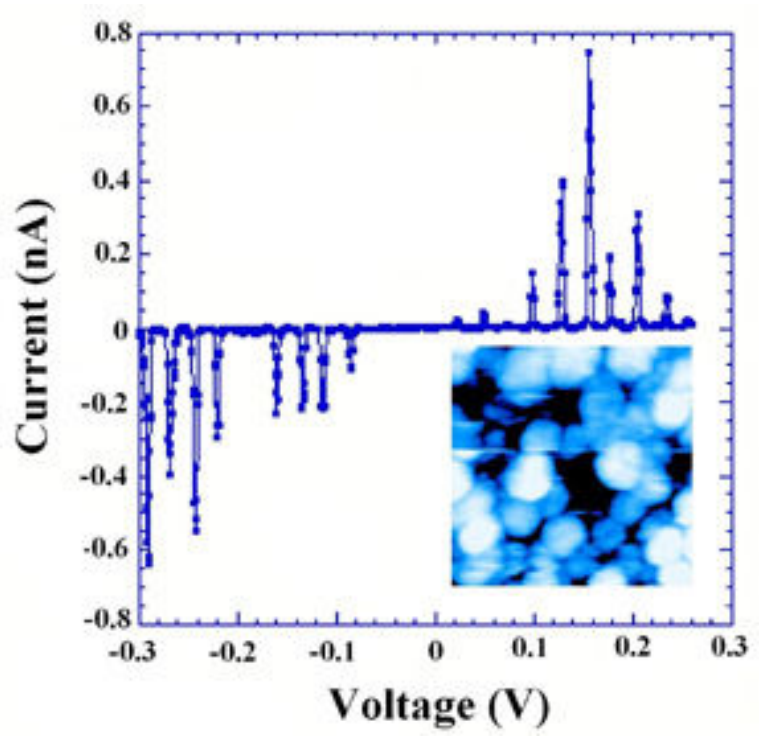

FIG. 2: Tunneling current versus voltage at $\mathrm{T}=4.2 \mathrm{~K}$. Tunneling is from a tunsten STM tip into a $\mathrm{Pb}$ cluster. Inset: $30.0 \mathrm{~nm} \times 30.0 \mathrm{~nm}$ image of $\mathrm{Pb}$ clusters grown by a buffer layer assisted growth technique. This image was obtained using a bias voltage of $-3.0 \mathrm{~V}$ with a tunneling current of $2.0 \mathrm{x}$ $10^{-9} \mathrm{~A}$.

\section{CURRENT-VOLTAGE CHARACTERISTICS}

In this section we shall be concerned with measurements of current-voltage characteristics of a series combination of two sets of discrete energy levels of systems separated by insulating barriers. Electron transmission through the clusters was probed by the STM tip in spectroscopy mode. The tunneling current was recorded as a function of applied sample bias voltage $V$ while the STM feedback loop was open. In this case, the tip was held at a fixed height above the cluster while the current-voltage characteristics were recorded. The data presented in this work were all obtained in voltage step sizes of $480 \mu \mathrm{V}$ or $612 \mu \mathrm{V}$ in a time frame $\geq 0.2 \mathrm{~ms}$ per point.

Due to resonant tunneling between the tip, the cluster, the interface state, and finally the Pt electrode, the $I-V$ characteristics exhibit peaks as shown in Fig. 2, These peaks are irregularly spaced and varied in height and are dependent on cluster size. The statistics of peak heights and spacings will be discussed in the next section (Sec. IV). Several hundred $I-V$ curves were obtained on each cluster and their character varied along a given cluster in a nonsystematic manner.

The magnitude of the differential conductance is an appropriate measure of whether or not the tunneling regime is the correct description of the transport. This will be addressed in this section along with a brief discussion of elastic and inelastic tunneling as it pertains to the linewidths. Also the relationship between the $I-V$ curves and the sizes of the clusters will be discussed. But first we will take up the issue of resonant tunneling in this system. The data is quite different from that which would be obtained in a geometry in which metallic atoms (or clusters) are deposited on an oxidized metallic surface and transmission is via nonresonant process.$\frac{3}{3}$

\section{A. Resonant Tunneling Mechanism}

The observation of peaks instead of steps strongly suggests that the transport through the cluster is due to resonant tunneling processes ${ }^{20}-23$ One possible explanation of how this occurs is the existence of an interface state between the $\mathrm{Pb}$ clusters and the $\mathrm{Si}$ substrate. It is known from photoemission experiments that such an interface exists between $\mathrm{Pb}$ and $\mathrm{Si}(111)$ and this interface state is nearly dispersionless. 24 While there are differences in resistivity between the Si substrate that was used in our study and the photoemission experiment, we assume that such a state exists.

In all the $I-V$ spectra, the tunneling current is suppressed around zero bias followed by a series of peaks at both negative and positive bias. The current is suppressed when the energy levels of the cluster are not in registry with the interface state. The double barrier tunnel junction that is realized by positioning the STM tip over a $\mathrm{Pb}$ cluster is highly asymmetric with the first tunneling barrier between the tip and the cluster and the second barrier between the cluster and the substrate. This asymmetry is manifested in the asymmetry of the $I-V$ curves about zero bias.

Resonance occurs when the Fermi wavelength spans the length of the cluster. The Fermi wavelength for $\mathrm{Pb}$ is $4 \AA$ at room temperature ${ }^{25}$ Given that the average height is $8 \AA$, and measurements were carried out at liquid helium temperatures, the assumption that the tunneling mechanism is a resonant process is appropriate.

\section{B. Level Spacing as a function of Cluster Size}

Table I catalogs the $\mathrm{Pb}$ clusters with the estimated number of atoms per cluster, and estimated and measured mean level spacings. The volume of the clusters was calculated assuming that each cluster is a hemispherical cap such that the volume, $\operatorname{Vol}$. is $\frac{\pi h}{6}\left(3 r^{2}+h^{2}\right)$, where $r$ is the radius and $h$ is the height of the cluster. Since the clusters were irregularly shaped, their radius was estimated from $r=\sqrt{\frac{l w}{\pi}}$ where $l$ is the length of the cluster and $w$ is the width. The estimated number of atoms was calculated from $\operatorname{Vol} . /\left(\frac{1}{4} a^{3}\right)$ where $a$ is the lattice parameter of $\mathrm{Pb}$ and is equal to $4.95 \AA .25$ The estimated mean level spacing, which was calculated using the nearly free electron model, is $\langle\Delta\rangle=\frac{2 \pi^{2} \hbar^{2}}{m k_{f} V o l}$ and compared to the measured mean level spacings. Several of the clusters' measured mean level spacing were in the range of 8-10 meV. There are several explanations for this. First, 
the majority of the clusters had the same height which is a result of the buffer layer assisted growth technique when using a four monolayer thick buffer layer. Second, the calculation of the volume is a rough estimate and not entirely accurate in that it oversimplifies the actual shape of the clusters. Third, the clusters were not well isolated from each other thus the width and the length could be larger if more than one cluster was taken into consideration. Also, tip convolution effects obscure the actual width and length of the clusters. Nonetheless there is an apparent dependency on the level spacing energy on the size of the clusters.

\section{Lineshapes and Differential Conductance}

Information about the intrinsic lifetime of the electronic states of a single metal cluster and the tunneling process (inelastic or elastic) is in part difficult to obtain because of the temperature of the reserviors. This can be circumvented by having two sets of electronic states separated by a barrier through which the current is measured. In this tunneling scenario, electron transport is expected to occur only when the energy levels of the two states are aligned with one another ${ }^{26}$ and the upper bounds of the lifetimes of energy levels of varying size clusters can be estimated from the average widths of the resonances that results from these level alignments. The reason that these widths are upper bounds is that they depend on both the reciprocals of the lifetimes of the electronic states and the tunneling rates. We fit the full width half maximum of the peak widths in the I$\mathrm{V}$ curves for three clusters to Lorentzians (Lorentzians provided the best fit to the data) and found that they varied depending on the cluster size. For cluster 1, the average peak width was $3.54 \mathrm{meV}$ (tunneling time: 1.9 $\times 10^{-13}$ seconds), for cluster 7 , the average peak width was $3.06 \mathrm{meV}$ (tunneling time: $2.4 \times 10^{-13}$ seconds) and for cluster 12 , the average peak width was $1.06 \mathrm{meV}$ (tunneling time: $6.2 \times 10^{-13}$ seconds) indicating that it takes longer to tunnel through a larger cluster than a smaller one. Lorentzian peak widths are also an indication that the tunneling process is elastic. Moreover, the absence of diffusion (the transport is ballistic) together with the spatial resolution of the STM enables detailed studies of the clusters' electronic states.

Figure 3 shows a plot of the differential conductance in units of $\mathrm{e}^{2} / \mathrm{h}$ plotted against positive bias for one of the clusters. This is supporting evidence that the features are related to elastic tunneling processes as the conductance is less than one and the peaks are Lorentzians.

\section{Position Space Representation of Eigenstates}

An important feature of the measurements was that the current-voltage characteristics varied with position on a cluster as shown in Fig. 4. The peak heights,

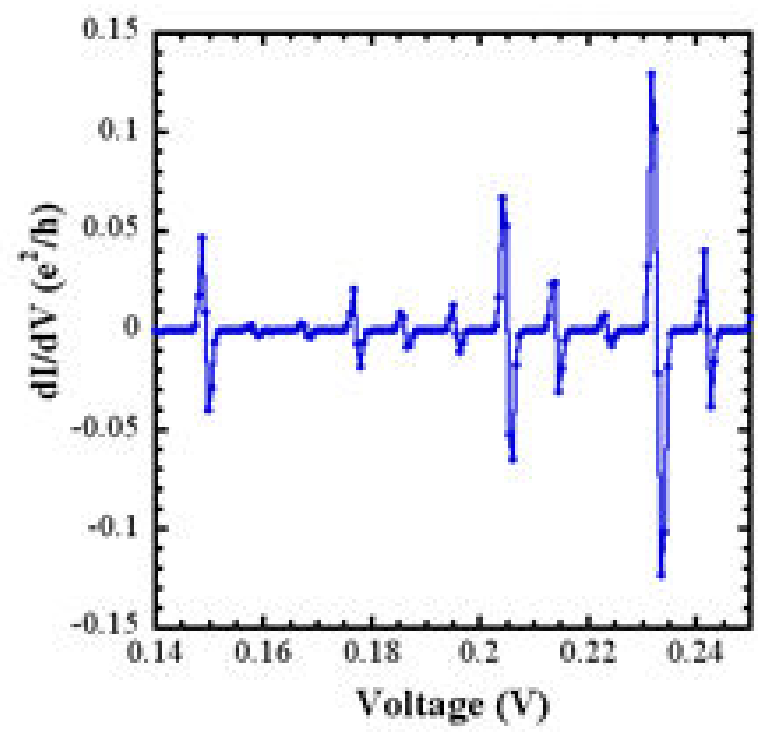

FIG. 3: Differential conductance (in terms of $\mathrm{e}^{2} / h$ ) versus voltage at $\mathrm{T}=4.2 \mathrm{~K}$ for cluster no. 12 . The symmetry about the $y$-axis and the magnitude of the peaks indicates that elastic tunneling processes are relevant.

the number of peaks, and voltages where the peaks occuried varied randomly as a function of position. This dependence is reminiscent of a quantum chaotic system in which the amplitude of the eigenfunctions are known to vary in a complicated pattern 27 There was no noticeable systematic variation in the curves as a function of distance. It was not possible to reproduce the curves because of the difficulty in repeating measurements at the exact same pixel point due to drift in the piezos which control the tip's position.

For a given current profile across a cluster, a map of the current as a function of the voltage bias can be obtained as shown in Fig. 5. For cluster no. 12, such a map displays strong clustering of current peaks as a function of applied bias voltage although there is no obvious voltage dependence.

\section{E. Other possible tunneling scenarios}

The current voltage characteristics bare a strikingly similar resemblance to the current vs gate voltage curves seen in the results reported by Kouwenhoven, Austing and Tarucha ${ }^{28}$ It might be the case, that neighboring clusters are actually gating the cluster under investigation. This would offer an alternative explanation to the position dependence of the I-V curves as the capacitance will change due to the distance between the gating cluster and the site on the cluster that was being probed by the STM tip. Further work is needed to elucidate the mechanism behind the peaks in the current-voltage characteristics. One such possible experiment would be to 


\begin{tabular}{|c|c|c|c|c|c|c|}
\hline $\begin{array}{c}\text { Cluster } \\
\text { Index No. }\end{array}$ & $\begin{array}{c}\text { Length } \\
(\mathrm{nm})\end{array}$ & $\begin{array}{c}V o l \\
(\mathrm{~nm})^{3}\end{array}$ & $\langle\mathrm{~N}>$ & $\begin{array}{c}\text { Est. }<\Delta> \\
(\mathrm{meV})\end{array}$ & $\begin{array}{c}\text { Meas. }<\Delta> \\
(\mathrm{meV})\end{array}$ & $\begin{array}{c}\partial E \partial \tau_{S O} \\
(\hbar=1)\end{array}$ \\
\hline 1 & 2.7 & 2.6 & 85 & 36.8 & $24.2 \pm 5.39$ & .083 \\
\hline 2 & 3.32 & 3.81 & 125 & 25.1 & $6.35 \pm 0.74$ & .070 \\
\hline 3 & 2.29 & 3.86 & 127 & 24.76 & $9.02 \pm 1.45$ & .047 \\
\hline 4 & 3.61 & 6.29 & 207 & 15.2 & $10.37 \pm 0.43$ & .046 \\
\hline 5 & 3.13 & 6.3 & 208 & 15.15 & $9.41 \pm 1.12$ & .04 \\
\hline 6 & 3.04 & 7.36 & 243 & 12.99 & $9.13 \pm 0.29$ & .033 \\
\hline 7 & 2.7 & 7.4 & 244 & 12.91 & $10.8 \pm 0.54$ & .029 \\
\hline 8 & 3.88 & 7.8 & 257 & 12.25 & $8.39 \pm 0.28$ & .04 \\
\hline 9 & 5.0 & 8.2 & 270 & 11.65 & $9.01 \pm 0.26$ & .049 \\
\hline 10 & 3.73 & 9.62 & 317 & 9.93 & $8.58 \pm 0.34$ & .031 \\
\hline 11 & 3.12 & 10 & 329 & 9.55 & $5.37 \pm 1.53$ & .025 \\
\hline 12 & 4.3 & 12.7 & 418 & 7.52 & $9.6 \pm 1.25$ & .027 \\
\hline 13 & 2.54 & 13.2 & 435 & 7.23 & $8.98 \pm 1.63$ & .0154 \\
\hline 14 & 5.49 & 19.4 & 638 & 4.94 & $7.35 \pm 1.30$ & .023 \\
\hline 15 & 4.12 & 21.5 & 709 & 4.44 & $7.49 \pm 0.37$ & .015 \\
\hline 16 & 4.76 & 27.1 & 892 & 3.53 & $4.73 \pm .87$ & .014 \\
\hline
\end{tabular}

TABLE I: Summary of physical parameters of Pb clusters of different shapes and sizes. As discussed in the text, the Vol. is the volume of a cluster and is estimated assuming it is a hemispherical cap. The number of atoms, $j \mathrm{~N}_{i}$, and the est. mean level spacing are calculated from this est. volume. The est. mean level spacing is based on the nearly free electron model and compared to experimentally measured mean level spacing values. The expression $\partial E \partial \tau_{S O}$ is related to the cluster's size and is described in the text (see Sec. IV D).

fabricate the clusters without the electrodes configured on top of the substrate. This would provide a more uniform distribution of clusters and increase the separation between clusters since the clusters tended to conglomerate near the electrodes.

\section{STATISTICAL DISTRIBUTIONS}

Current peaks in the scanning tunneling spectroscopy were not expected. However, in an attempt to interpret the results the data was analyzed with the assumption that the peaks were signatures of discrete energy levels. Exact agreement with different sets of discrete energy levels for both the negative and positive bias voltages would demand that the capacitances between both the tip and cluster and cluster and substrate are substantially different, which we believe is the case. Regardless, from the I-V measurements themselves, it is not possible to confirm that the peaks in the negative and bias voltages arise from different energy levels without further analysis. Therefore, based on the premise that the discrete energy spectra in metallic clusters should follow RMT, we studied the statistical distributions of both the peak spacings and peak heights. Peaks in the I-V characteristics were identified after running a smoothing program ${ }^{29}$ twice through the data. This program was used to identify all the peaks. Since the voltage range over which peaks were found in this study was small $(-35 \mathrm{meV}$ to $+30 \mathrm{meV}$ ), the likelihood of missing any peaks was elim- inated. A practical matter connected with the reliability of the histograms must also be mentioned. The number of bins used in the histograms was the range divided by the experimental step size. The histograms were rescaled using the measured mean level spacing to normalize the mean to unity. The fits to the resulting histogram were carried out using MINOS30 which is a minimization algorithm implemented in MINUIT 31 The parameters that are obtained are those that correspond to a minimum chisquared value. Also, the peak widths are approximately ten times greater than the bin size, thus the peaks spacing distribution that was generated is not due to a "binning" effect.

\section{A. Wigner-Dyson Statistics}

The histogram of normalized peak spacings (normalized to the mean spacing of each individual trace) was fit by the distribution function

$$
P(s)=b_{\beta} s^{\beta} \exp \left(-a_{\beta} s^{c_{\beta}}\right)
$$

The distribution function $\mathrm{P}(\mathrm{s})$ is a probability density defined such that the area under the curve is one. Here the normalized mean spacing, $s$, is simply $\Delta /\langle\Delta\rangle$, with $\Delta$ representing the level spacing and $\langle\Delta\rangle$ the mean spacing. Equation 1 can represent the orthogonal $(\beta=1)$, unitary $(\beta=2)$ and symplectic $(\beta=4)$ ensembles 32 that correspond to processes with different symmetries. 


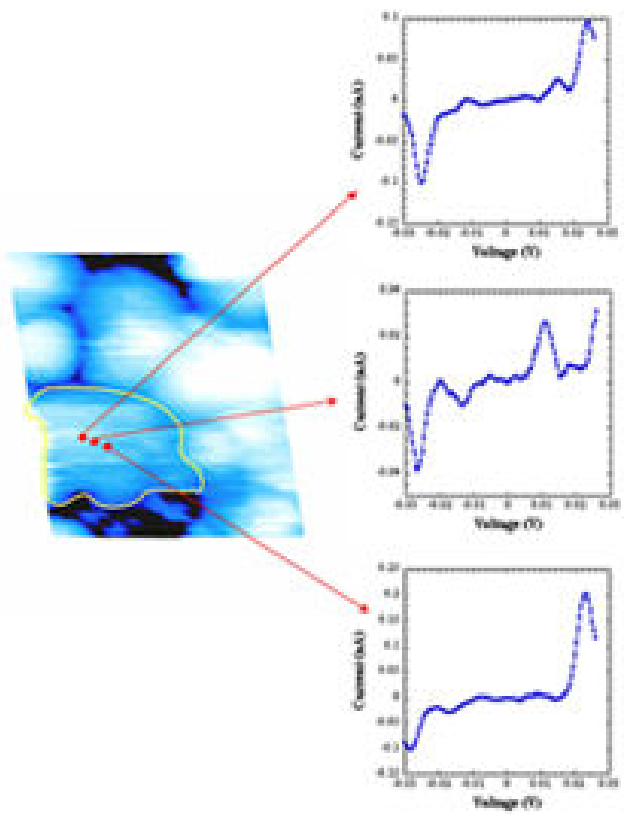

FIG. 4: STM image $(12 \mathrm{~nm} \times 8.0 \mathrm{~nm})$ of $\mathrm{Pb}$ clusters. The $I-V$ curves correspond to three different positions along a cluster. There is not any apparent correlation between the curves. The current setpoint $=1.0 \mathrm{nA}$.

The orthogonal case corresponds to time reversal symmetry being preserved in the absence of a magnetic field and describes the results presented here. In the statistical analysis of this histogram fits by Wigner-Dyson, Poisson, Gaussian, and Lorentzian distributions were made. From the values of $\chi^{2}$ (not shown) it is clear that the Wigner-Dyson distribution provides the best fit to the data with $a_{\beta}=\pi / 4, b_{\beta}=\pi / 2$ and $c_{\beta}=2$. In Fig. [6] the histogram of peak spacings for this cluster, showing the Wigner-Dyson and Poisson fits is plotted. (In the figure, the fitted Poisson distribution is not one about the origin since the parameters were allowed to float in order to minimize $\operatorname{chi}^{\wedge} 2$ ).

\section{B. Porter-Thomas Statistics}

The following form ${ }^{33}$,

$$
P(I)=a\left(\frac{I}{<I>}\right)^{b} \exp \left[-c\left(\frac{I}{<I>}\right)\right]
$$

was fit to the data, where $I$ is the peak current and $<I>$ the mean peak current. In this analysis, parameters specific to the Porter-Thomas and Poisson distributions, which were deemed relevant, were used. The Porter-Thomas distribution (with $a=(2 \pi)^{-\frac{1}{2}}, b=-1 / 2$ and $c=1 / 2$ ) provided a somewhat better fit to the data than the Poisson distribution. Figure 7 shows a plot of the histogram along with curves associated with the best fits of the Porter-Thomas and Poisson distributions. The

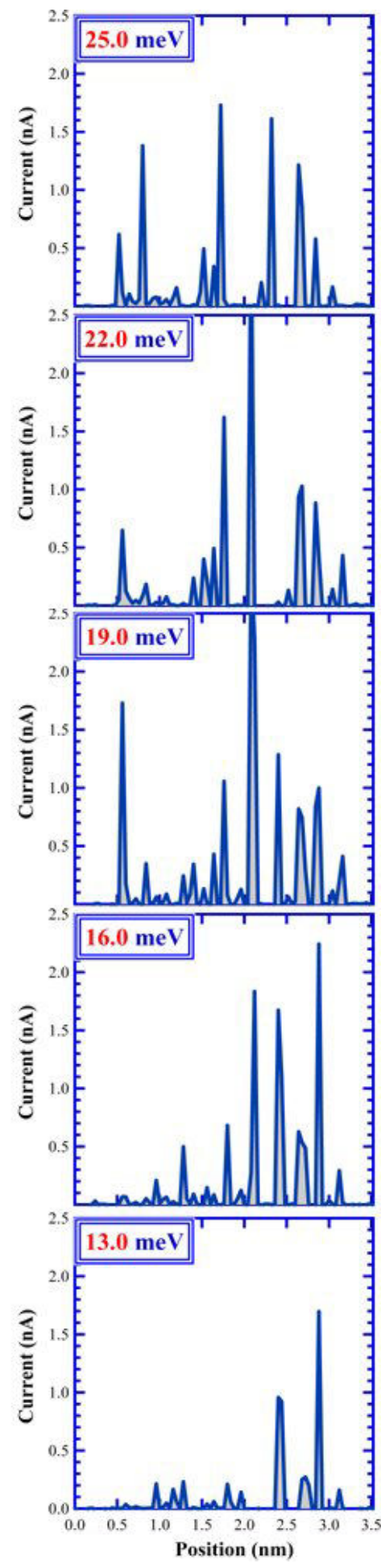

FIG. 5: A map of the current as a function of position along a chaotic cluster (catalog number 4) as the bias voltage is varied. There is noticable clustering of the current peaks. 


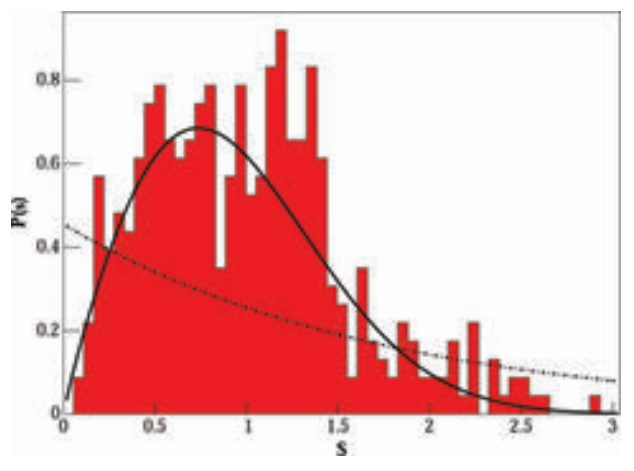

FIG. 6: Histogram of peak spacings for cluster no. 9. The solid curve is the fit for the Wigner-Dyson distribution. The dotted line represents the fit for the Poisson distribution. There are 413 peak spacings that comprise this histogram normalized to the mean voltage spacing.

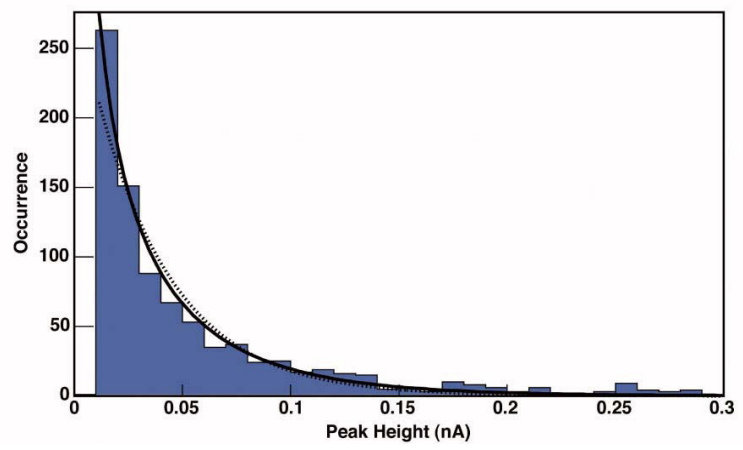

FIG. 7: Histogram of peak heights for cluster no. 9. The solid curve is the fit for the Porter-Thomas distribution. The dotted line represents the fit for the Poisson distribution. There are 851 peaks heights that comprise the histogram.

results of this analysis support the interpretation that these measurements are yielding spectroscopic information relating to the energy levels.

\section{Crossover between Poisson-like and Wigner- Dyson Statistics}

Experimentally one should observe variations in the distributions of the energy levels based on the cluster's shape. Keeping all the experimental parameters the same, i.e. the same tip-cluster height distance and the same voltage scale, the distributions of the eigenvalues change depending on the cluster's shape. In the work presented here, the majority of distributions for the different clusters fell into the regime between Wigner-Dyson and Poisson statistics, which describes a partially chaotic system. This is plotted in Fig. 8 where clusters of the same approximate volume but have different shapes are considered. The top histogram in Fig. 8 is more Poisson-like while the bottom histogram is strongly Wigner-Dyson like. This is in agreement with other experimental situations, such as the He atom in a magnetic field 15 and the acoustic resonances of $\mathrm{Al}$ blocks where deformations in the shape of the block away from parallelpiped generate partially chaotic acoustic waves ${ }^{34}$ Completely chaotic systems are rare and difficult to achieve experimentally.

The modified Berry-Robnik equation ${ }^{35}$ indicates what percentage of the distribution is Poisson like and what percentage is Wigner-Dyson like and accounts for the small level repulsion that is observed in most systems. (It is a modified version of the Berry-Robnik distribution ${ }^{36}$ which works well for mixed chaotic and regular systems at the tail of the distribution, but fails at small level spacings.). Thus the modified Berry-Robnik equation is

$$
\begin{aligned}
P(s) & \propto q^{2} \mathrm{~F}\left(\frac{s}{\nu^{2}}\right) \mathrm{e}^{-q s} \operatorname{erfc}\left(\frac{\sqrt{\pi}}{2}(1-q) s\right)+ \\
& +\left[\frac{\pi}{2}(1-q)^{2} s+2 q F\left(\frac{s}{\nu}\right)\right](1-q) e^{-q s-\frac{\pi}{4}(1-q)^{2} s^{2}}
\end{aligned}
$$

where erfc is the complimentary of the error function and is defined as

$$
\operatorname{erfc}(x)=1-\operatorname{erf}(x)=\frac{2}{\sqrt{\pi}} \int_{x}^{\infty} e^{-u^{2}} d u
$$

In addition, $F(x)$ is defined as follows:

$$
F(x)=1-\frac{1-\sqrt{\frac{\pi}{2}} x}{e^{x}-x}
$$

When $\mathrm{q}=0$ the Wigner-Dyson term is retrieved and when $\mathrm{q}=1$, the distribution is a Poisson distribution. Equation 3 describes a mixed state between these two limits and addresses the crossover regime in which the dynamics are partially chaotic.In Table III the fitted values for the modified Berry Robnik equations are summarized for different sized $\mathrm{Pb}$ clusters.

\section{Absence of Charging Energy, Superconductiv- ity and Spin-Orbit Scattering}

An important issue is the role of charging energy in the proposed two-step tunneling process. It is likely that the charging energy is absent because of additional capacitance arising from the proximity of neighboring clusters. This will lower the charging energy significantly so that the peaks that are observed are only those related to the eigenstates of the clusters. Absence of charging energy was also recently reported from x-ray photoemission spectroscopy measurements 37 on gold nanocrystals which were self-assembled using wet chemistry. In this study, as the mutual separation between gold clusters 

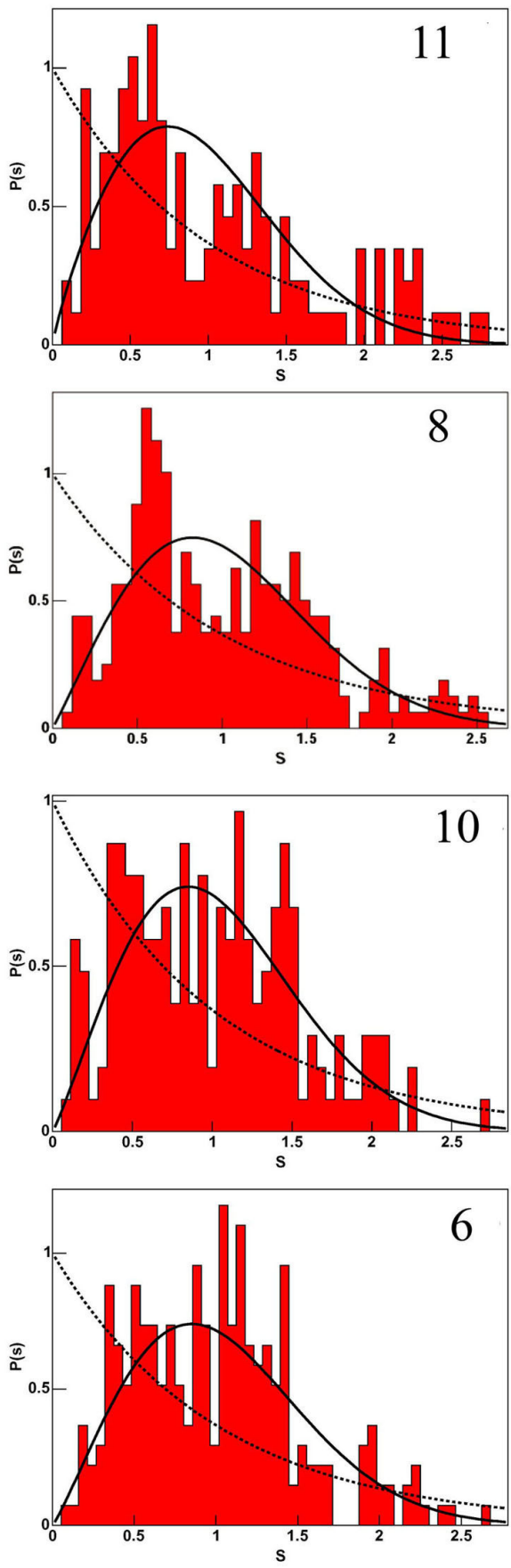

FIG. 8: Transition to chaos in the level statistics of $\mathrm{Pb}$ clusters of the same approximate volume $\left(\approx 10 \mathrm{~nm}^{3}\right)$ but with different shapes. The solid black curve is the Wigner-Dyson distribution, while the dotted black curve is the Poisson distribution for comparison. The cluster's catalog number is in the upper right hand corner.

\begin{tabular}{|c|c|c|c|c|}
\hline Cluster & $\begin{array}{c}\text { \# I-V } \\
\text { curves }\end{array}$ & $\begin{array}{c}\text { \# Peak } \\
\text { Spacings }\end{array}$ & $\mathrm{q}$ & $\nu$ \\
\hline 1 & $<10$ & $<30$ & - & - \\
\hline 2 & 180 & 35 & - & - \\
\hline 3 & 119 & 137 & $.59 \pm 0.71$ & $.65 \pm 0.22$ \\
\hline 4 & 137 & 144 & $0.02 \pm 0.06$ & - \\
\hline 5 & 125 & 16 & - & - \\
\hline 6 & 158 & 254 & $-3.1 \mathrm{e}-9 \pm 4.39 e-02$ & - \\
\hline 7 & $<10$ & $<30$ & - & - \\
\hline 8 & 207 & 273 & $.14 \pm 0.085$ & $.45 \pm 0.17$ \\
\hline 9 & 280 & 413 & $0.02 \pm 0.037$ & - \\
\hline 10 & 131 & 181 & $.06 \pm .062$ & $.18 \pm 0.25$ \\
\hline 11 & 316 & 142 & $1.37 \pm 0.56$ & $.73 \pm 0.06$ \\
\hline 12 & $<10$ & $<30$ & & - \\
\hline 13 & 193 & 237 & $0.004 \pm 0.05$ & - \\
\hline 14 & 405 & 64 & $1.28 \pm 0.58$ & $.7 \pm 0.16$ \\
\hline 15 & 220 & 105 & $-0.0086 \pm .07$ & - \\
\hline 16 & 263 & 450 & $0.25 \pm 0.07$ & $.48 \pm .08$ \\
\hline
\end{tabular}

TABLE II: Summary of the statistics of different sized $\mathrm{Pb}$ clusters. When $q=0$ or close to it, WD statistics prevails and the value of $\nu$ becomes meaningless, and when $\mathrm{q}=1$ Poisson statistics is the appropriate description of the distribution. (The low statistics for cluster numbers 1, 2, 5, 7, and 12 resulted in undefined $\mathrm{q}$ and $\nu$ values.)

was reduced to distances less than $1 \mathrm{~nm}$ by varying the ligand size, the charging energy was eliminated. New experimental conditions need to be realized in our work in which the clusters are well separated from each other in order to resolve this issue about the charging energy.

The apparent absence of superconductivity is a result of the mean level spacing exceeding the energy gap of superconducting $\mathrm{Pb}$ which is $\sim 2 \mathrm{meV}$. For this reason one would not expect to observe features in the tunneling characteristic associated with superconductivity $\underline{38}$ This was also observed in the pioneering work by Ralph, Black and Tinkham $\cdot \underline{3}$

Lead is a strong spin-orbit coupling material and the appropriate description to describe strong spin orbit coupling is a Gaussian Sympletic Ensemble (GSE) instead of Gaussian Orthogonal Ensemble (GOE or Wigner-Dyson) distribution. How strongly spin orbit coupling adheres to GSE instead of GOE can be related to the cluster's size ${ }^{39}$ as follows: If Wigner-Dyson (GOE) statistics prevail, then the following criteria based on the uncertainity principle $(\hbar=1)$ should be met: $\delta E \delta \tau_{S O} \gg 1$. Likewise, if the appropriate statistics is related to Gaussian Sympletic Ensemble (GSE), then the following criteria is applicable: $\delta E \delta \tau_{S O} \ll 1$. In these relations, $\delta E$ is the mean energy level spacing, or one divided by the density of states. The term $\delta \tau_{S O}$ is the time that it takes to flip a spin or $\delta \tau_{S O}=\frac{L}{v_{f}}$ where $L$ is the length of the cluster, and $v_{f}$ is the Fermi velocity. This assumes that the lateral transport in the dot is ballistic with the only 


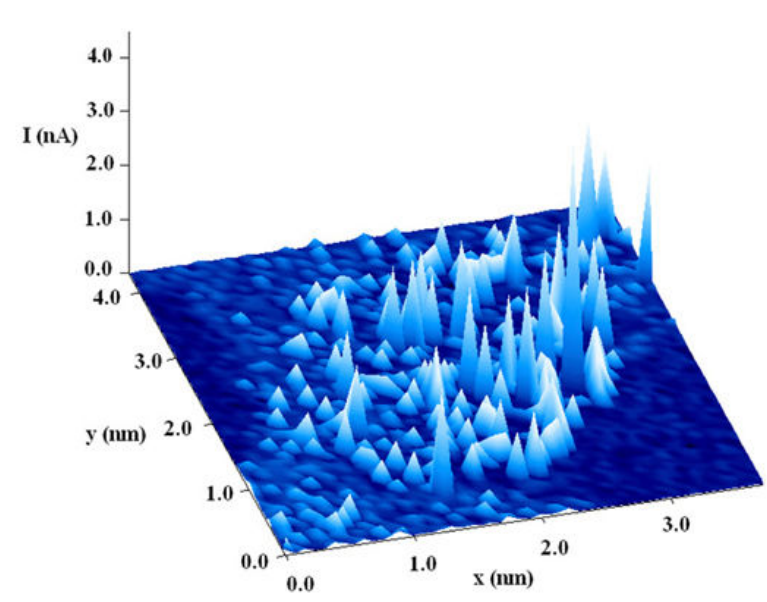

FIG. 9: Current as a function of position on a chaotic cluster (cluster no. 4) at $26 \mathrm{meV}$.

scattering occuring at the boundaries. Thus the expression $\delta E \delta \tau_{S O}$ is equal to $\frac{2 \pi^{2} L}{k_{f}^{2} V o l}$. In the Table $\prod$ the values for $\delta E \delta \tau_{S O}$ are calculated based on cluster size instead of the actual mean level spacing which is smaller than theoretical estimates. This upper limit indicates that the appropriate distribution should be GSE instead of GOE. This contradicts our experimental findings.

It should be noted that in studies of $\mathrm{Au}$ grains $(<5$ $\mathrm{nm}$ ), it was found that the spin-orbit scattering was suppressed $\stackrel{40}{ }$ It was speculated that the origin of the suppression of the spin-orbit scattering was the granularity of the weakly coupled grains. Estimates of this suppression were carried out by making weak localization measurements. This involves a study of the orbital effect in an applied magnetic field. While we could not make such estimates (our apparatus lacks a magnetic field) using similar arguments, we may have observed a suppression of spin-orbit scattering (based on the statistics).

\section{IMAGING QUANTUM CHAOS WITH AN STM}

Finally we turn our attention to imaging the eigenfunctions of the clusters using a scanning tunneling microscope. The wavefunction moduli are directly related to the tunneling current, so by mapping the tunneling current amplitude at a fixed bias, it is possible to study the significance of chaos as a function of cluster shape. An image of the current peaks is shown in Fig. 9 for cluster no. 4. The current peaks change as a function of applied bias (not shown).

The current dependence as a function of bias voltage can be used to "image" the chaotic nature of irregularly shaped metallic clusters. This gained prominence in the experimental community with the pioneering experiments of Sridhar and co-workers $\stackrel{41}{ }$ Besides acquiring images of the clusters, it is possible to produce images of chaotic behavior in the same manner than was carried out by Sridhar using electric field to probe the distributions of eigenfunctions in a "Sinai and rectangular" structure. In essence scanning tunneling spectroscopy is a technique that can be used to map out the amplitude of the eigenfunctions which are otherwise difficult to access.

\section{CONCLUDING REMARKS AND OPEN QUESTIONS}

The empirical evidence indicates that discrete energy levels are being accessed from a resonant tunneling geometry and that the distributions of these energy levels reflect the underlying classical dynamical nature of an electron under confinement of its boundary. This means that our study of the distribution of spacings gives information about the symmetry properties of the physical system in question. In this study of metal clusters, there are remaining questions that still need be resolved. First what is the nature of the tunneling process? Is the electron's route through an Si interface state, or through neighboring clusters? Future experimental work needs to be carried out in which the clusters are separated from one another in order to resolve this issue. The second question that needs to be answered is why are the spinorbit interactions suppressed? That is, why not Gaussian Sympletic Ensemble statistics instead of WignerDyson statistics? This issue needs to be explored both theoretically and experimentally in greater detail.

\section{ACKNOWLEDGMENTS}

It is a pleasure to thank the experts in this field that have provided useful and stimulating discussions with us about our work. Specifically we thank Leonid Glazman, Alex Kamenev, and Denis Ullmo. Also, one of us (L. L. A. A.) would like to thank the organizers, instructors and participants attending the Boulder Condensed Matter Summer School (2005) for many lively and helpful discussions. This work was supported by the US Department of Energy under grant DE-FG02-02ER46004.
1 M. L. Mehta, Random Matrices, 2nd ed., (Academic Press, New York, (1967)); W. P. Halperin, Rev. Mod. Phys. 58, 533 (1986); T. A. Brody, J. Flores, J. B. French, P. A. Mello, A. Pandey and S. S. M. Wong, Rev. Mod. Phys. 53, 385 (1981); A. D. Mirlin, Phys. Rep. 326, 259 (2000);
Y. Alhassid, Rev. Mod. Phys. 72, 895 (2000).

2 J. B. Garg, J. Rainwater, J. S. Petersen, and W. W. Havens, Jr., Phys. Rev. 134, B985 (1964).

3 D. C. Ralph, C. T. Black and M. Tinkham, Phys. Rev. Lett. 74, 3241 (1995). 
4 G. A. Narvaez and G. Kirczenow, Phys. Rev. B. 65, 121403 (2002).

${ }^{5}$ K. B. Efetov, Adv. Phys. 32, 53 (1983).

6 O. Agam, N. S. Wingreen, B. L. Altshuler, D. C. Ralph and M. Tinkham, Phys. Rev. Lett. 78, 1956 (1997).

7 B. Wang, H. Wang, H. Li, C. Zeng, J. G. Hou and X. Xiao., Phys. Rev. B 63, 035403-1 (2000).

${ }^{8}$ U. Banin, Y. Cao, D. Katz and O. Millo, Nature 400, 542 (1999).

9 E. P. A. M. Bakkers, Z. Hens, L. P. Kouwenhoven, L. Gurevich and D. Vanmaekelbergh, Nanotechnology 13, 258 (2002).

10 U. Sivan, R. Berkovits, Y. Aloni, O. Prus, A. Auerbach and G. Ben-Yoseph, Phys. Rev. Lett. 77, 1123 (1996); F. Simmel, T. Heinzel and D. A. Wharam, Europhys. Lett., 38, 123 (1997); S. Patel, S. M. Cronenwett, D. R. Stewart, A. G. Huibers, C. M. Marcus, C. I. Duruoz, J. S. Harris, Jr., K. Campman and A. C. Gossard, Phys. Rev. Lett. 80, 4522 (1998); C. M. Marcus, S. R. Patel, C. I. Duruoz, J. S. Harris, K. Campman and A. C. Gossard, Physica B 249, 201 (1998); F. Simmel, D. Abusch-Magder, D. A. Wharam, M. A. Kastner and J. P. Kotthaus, Phys. Rev. B. 59, 10441 (1999).

11 O. Bohigas, M. J. Giannoni and C. Schmit, Phys. Rev. Lett. 52, 1 (1984)..

12 Wigner, in Proc. Canadian Mathematical Congress, (Univ. of Toronto Press, Toronto, 1957); F. J. Dyson, J. Math. Phys. 3, 140 (1962).

13 C. E. Porter, Statistical Theories of Spectra: Fluctuations, (Academic, New York 1965); R. G. Thomas and C. E. Porter, Phys. Rev. 104, 483 (1956).

14 T. S. Monteiro and G. Wunner, Phys. Rev. Lett. 65, 2360 (2000).

15 K. Karremans, W. Vassen and W. Hogervorst, Phys. Rev. Lett. 81, 4843 (1998).

16 L. L. A. Adams, B. W. Lang, and A. M. Goldman, Phys. Rev. Lett. 95, 146804 (2005).

17 Virginia Semiconductor Inc., 1501 Powhalan St., Fredericksburg, VA 22401 USA.

${ }^{18}$ L. Huang, S. J. Chey and J. H. Weaver, Phys. Rev. Lett. 80, 4095 (1998).

19 L. L. A. Adams and A. M. Goldman, Rev. Sci. Instrum. 76, 063907 (2005).

${ }^{20}$ L. L. Chang, L. Esaki, and R. Tsu, Appl. Phys. Lett. 24, 593 (1974).

21 N. C. van der Vaart, S. F. Godijn, Y. V. Nazarov, C. J. P. M. Harmans, J. E. Mooij, L. W. Molenkamp and C. T. Foxon, Phys. Rev. Lett. 74, 4702 (1995).

22 M. Tewordt, H. Asahi, V. J. Law, R. T. Syme, M. J. Kelly, D. A. Ritchie, A. Churchill, J. E. F. Frost, R. H. Hughes and G. A. C. Jones, Appl. Phys. Lett. 60, 595 (1992).

23 P. B. Wilkinson, T. M. Fromhold, L. Eaves, F. W. Sheard, N. Miura and T. Takamasu, Nature 380, 608 (1996).

${ }^{24}$ H. H. Weitering, A. R. H. F. Ettema and T. Hibma, Phys. Rev. B 45, 9126 (1992).

25 C. Kittel, Introduction to Solid State Physics, $7^{\text {th }}$ Edition, p. 150, John Wiley \& Sons, Inc., (1996).

26 Y. V. Nazarov, Physica B 189, 57 (1993).

27 Arnd Bäcker, preprint, cond-mat/nlin.CD/0204061.

28 L. P. Kouwenhoven, D. G. Austing and S. Tarucha, Rep. Prog. Phys. 64, 701 (2001).

29 Details for the smoothing program can be found in Jerome H. Friedman's report, CERN School of Computing Proceedings, 3rd, Godoysund, Norway
(1974), Yellow Report 74-23. This can be found at: http://www.slac.stanford.edu/cgi-wrap/getdoc/slac-r-176.pdf

30 http://root.cern.ch/

31 http://www.dnp.fmph.uniba.sk/cernlib/asdoc/minuit/minmain.html

32 D. A. Rabson, B. N. Narozhny and A. J. Millis, Phys. Rev. B 69, 054403-1 (2004).

33 E. Runge and R. Zimmermann, Phys. Stat. Sol. (B) 221, 269 (2000).

34 C. Ellegaard, T. Guhr, K. Lindemann, H. Q. Lorensen, J. Nygard, M. Oxborrow, Phys. Rev. Lett. 75, 1546 (1995).

35 Viktor A. Podolskiy and Evgenii E. Narimanov, preprint, cond-mat 0310034 (2003).

36 M. V. Berry, M. Robnik, J. of Physics A, 17, 2413 (1984).

37 H. Liu, B. S. Mun, G. Thornton, S. R. Isaacs, Y-S. Shon, D. F. Ogletree, and M. Salmeron, Phys. Rev. B 72, 155430 (2005).

38 P. W. Anderson, J. Phys. Chem. Solids 11, 26 (1959).

${ }^{39}$ K. A. Matveev, L. I. Glazman and A. I. Larkin, Phys. Rev. Lett. 85, 2789 (2000).

40 A. Anaya, M. Bowman and D. Davidovic, Phys. Rev. Lett. 93, 246604 (2004).

41 Kudrolli, V. Kidambi, and S. Sridhar, Phys. Rev. Lett. 75, 822 (1995); P. Pradhan and S. Sridhar, Phys. Rev. Lett. 85, 2360 (2000). 